\title{
ON THE SHARPNESS OF $L^{2}$-ERROR ESTIMATES OF $H_{0}^{1}$-PROJECTIONS ONTO SUBSPACES OF PIECEWISE, HIGH-ORDER POLYNOMIALS
}

\author{
WEIMIN HAN AND SØREN JENSEN
}

Dedicated to Professor R. Bruce Kellogg on the occasion of his 60th birthday 1

\begin{abstract}
In a plane polygonal domain, consider a Poisson problem $-\Delta u=$ $f$ with homogeneous Dirichlet boundary condition and the $p$-version finite element solutions of this. We give various upper and lower bounds for the error measured in $L^{2}$. In the case of a single element (i.e., a convex domain), we reduce the question of sharpness of these estimates to the behavior of a certain inf-sup constant, which is numerically determined, and a likely sharp estimate is then conjectured. This is confirmed during a series of numerical experiments also for the case of a reentrant corner. For a one-dimensional analogue problem (of rotational symmetry), sharp $L^{2}$-error estimates are proven directly and via an extension of the classical duality argument. Here, we give sharp $L^{\infty}$-error estimates in some weighted and unweighted norms also.
\end{abstract}

\section{INTRODUCTION}

The purpose of the paper is to study the influence of corner singularities on the accuracy of $p$-version finite element solutions, and the sharpness of $L^{2}$-norm error estimates. We note that in the context of the $h$-version finite element method for a corner problem, the sharpness of $L^{2}$-norm error estimates, and more generally of error estimates in negative-order Sobolev norms, has been fully studied in [17]. Some attempts have also been made regarding the $p$-version of the finite element method. In [13], error estimates in $L^{2}$ and $H^{-s}(s>0)$ are derived, using the traditional duality technique. However, the question of the sharpness of $L^{2}$-norm error estimates is unanswered. This paper is a further attempt to answer the question. In $\S 2$, we give an $L^{2}$-norm error bound. In $\S 3$, we derive a lower bound for the $L^{2}$-norm error. By connecting to the optimality constant, the stability constant and inf-sup constants, we conjecture a likely optimal $L^{2}$-norm error estimate. The conjectured optimal $L^{2}$-norm error estimate is proved in $\S 4$ in a one-dimensional setting which mimics the two-dimensional corner singularities. The one-dimensional singular

Received by the editor June 18, 1993 and, in revised form, February 17, 1994.

1991 Mathematics Subject Classification. Primary 65N15, 65N30.

${ }^{1}$ These results were presented at the Finite Element Circus held in his honor at Pennsylvania State University on November 9, 1991.

This work was partially supported by the Old Gold Fellowship of the University of Iowa (W.H.) and by ONR grant N00014-90-J-1238 (S.J.). 
model problem we study is in some sense closer to two-dimensional corner problems than the one considered in [8]. In $\S 5$, we prove various sharp $L^{\infty}$-norm error estimates for the one-dimensional model problem. In the last section, we present numerical results on corner domains with various internal angles to confirm the likely optimal $L^{2}$-norm error estimate.

Let $\Omega$ be a bounded, simply connected polygonal domain in the plane. Consider the Dirichlet boundary value problem

$$
\left\{\begin{aligned}
-\Delta u=f & \text { in } \Omega, \\
u=0 & \text { on } \partial \boldsymbol{\Omega}
\end{aligned}\right.
$$

with $f \in H^{s-1}(\Omega)$ for some $s \geq 0$. Let $\omega_{1} \leq \omega_{2} \leq \cdots \leq \omega_{M-1} \leq \omega_{M}$ denote the interior angles at the corners $A_{j}$ of $\Omega, 1 \leq j \leq M$. Let

$$
\alpha_{j}=\frac{\pi}{\omega_{j}} \in\left[\frac{1}{2}, \infty\right), \quad 1 \leq j \leq M .
$$

The regularity of the solution $u$, in the presence of corners, is the subject of some classical treatments, cf. Dauge et al. [5], Grisvard [10], Kellogg [14] and Kondrat'ev [15]. Following Dauge et al., we introduce singular functions, written in terms of polar coordinates, centered at the vertex of a cone $\Gamma$. Within a neighborhood of a corner $A_{j}$, the domain $\Omega$ coincides with a cone $\Gamma_{j}=$ $\left\{\left(r_{j}, \theta_{j}\right): 0<r_{j}<\infty, 0<\theta_{j}<\omega_{j}\right\}$. Then, the singular functions are

$$
S_{j, l}=\eta_{j} \sigma_{j, l}, \quad l \in Z_{+},
$$

where $\eta_{j} \in C^{\infty}$ is a cutoff function, $\eta_{j}=1$ near $A_{j}$, and $\eta_{j}=0$ outside some neighborhood of $A_{j}$,

$$
\sigma_{j, l}=\left\{\begin{array}{l}
r_{j}^{\lambda_{j, l}} \sin \left(\lambda_{j, l} \theta_{j}\right) \quad \text { if } \lambda_{j, l} \notin Z_{+}, \\
r_{j}^{\lambda_{j, l}}\left(\sin \left(\lambda_{j, l} \theta_{j}\right) \log r_{j}+\theta_{j} \cos \left(\lambda_{j, l} \theta_{j}\right)\right) \quad \text { if } \lambda_{j, l} \in Z_{+}
\end{array}\right.
$$

and

$$
\lambda_{j, l}=l \alpha_{j}=\frac{l \pi}{\omega_{j}} .
$$

Then we can write the solution to the problem (1.1) as follows: supposing $f \in H^{s-1}(\Omega), s \in R_{+} \backslash Z$, we have

$$
u=u_{0}+\sum_{j=1}^{M} \sum_{\lambda_{j, l}<s} \gamma_{j, l} S_{j, l}
$$

with

$$
u_{0} \in H^{s+1}(\Omega) \text { and }\left\|u_{0}\right\|_{s+1}+\sum_{j, l}\left|\gamma_{j, l}\right| \leq c\left(\|f\|_{s-1}+\|u\|_{1}\right) .
$$

The singular expansion coefficients $\gamma_{j, l}$ are exhibited as linear functionals of $f$ in $[5, \S 5]$. The leading singularity in (1.3) is $S_{M, 1} \in H^{1+\alpha_{M}-\varepsilon}(\Omega), \forall \varepsilon>0$. We denote

$$
\alpha \stackrel{\text { def }}{=} \alpha_{M} \text {. }
$$




\section{UPPER $L^{2}$-ERROR BOUNDS}

Partition $\Omega$ into $E$ elements $\bar{\Omega}=\bigcup_{i=1}^{E} \bar{\Omega}_{i}$, where $\Omega_{i}$ are parallelograms or triangles with $\boldsymbol{\Omega}_{i} \cap \boldsymbol{\Omega}_{j}=\varnothing$ for $i \neq j$ and $\boldsymbol{\Omega}_{i} \cap \boldsymbol{\Omega}_{j}$ is either empty, a vertex or a common side of both $\Omega_{i}$ and $\Omega_{j}$. Each vertex of $\Omega$ is assumed to be a vertex of some $\Omega_{i}$. We denote by $\mathscr{P}$ the set of parallelograms and by $\mathscr{T}$ the set of triangles in the Euclidean plane.

Let $R=(-1,1)^{2}$ and $T=\{(x, y):-1<y<x,|x|<1\}$ denote the standard square and triangle, respectively. Let $F_{i}$ be an affine, orientation preserving (i.e., $\operatorname{det}\left(D F_{i}\right)>0$ ) mapping which maps $\Omega_{i}$ onto $R$ if $\Omega_{i}$ is a parallelogram, and onto $T$ if $\Omega_{i}$ is a triangle. Then we define the space of piecewise polynomials

$$
S^{p}=\left\{v \in H_{0}^{1}(\Omega):\left.v\right|_{\Omega_{i}} \circ F_{i}^{-1} \in\left\{\begin{array}{ll}
Q_{p}(R) & \text { if } \Omega_{i} \in \mathscr{P} \\
P_{p}(T) & \text { if } \Omega_{i} \in \mathscr{T}
\end{array}\right\},\right.
$$

where $Q_{p}$ and $P_{p}$ denote the spaces of polynomials of separate degree, respectively total degree, less than or equal to $p$.

The Galerkin solution $u_{p} \in S^{p}$, characterized by varying $p$ (and fixed partition), is then defined by

$$
\int_{\Omega} \nabla u_{p} \nabla v d x=\int_{\Omega} f v d x, \quad \forall v \in S^{p},
$$

or we may write $u_{p}=\Pi_{p} u$, where $\Pi_{p}$ is the $H_{0}^{1}$-projection onto $S^{p}$ (defined by the Dirichlet form in (2.2)).

It is well known, cf. [3], that, denoting by $e_{p}=u-u_{p}$, one has

$$
\left\|e_{p}\right\|_{1} \leq c p^{-\min (2 \alpha, s)}
$$

and that, in general, one cannot expect a better convergence rate than that in the estimate (2.3). In this paper, we are interested in the $L^{2}$-norm error estimate.

Proposition 2.1 (as in [13, Theorem 2]). Let $f \in H^{s-1}(\Omega), s \in R_{+} \backslash Z$. Assume $\alpha=\alpha_{M} \notin Z$. Let $u_{p}=\Pi_{p} u$ be defined as above. Then

$$
\left\|e_{p}\right\|_{0} \leq c p^{-\min (s+1,2 \alpha+1)} \text {. }
$$

Proof. Define an auxiliary function $w_{p} \in H_{0}^{1}(\Omega)$ by

$$
\left\{\begin{aligned}
-\Delta w_{p}=e_{p} & & \text { in } \Omega, \\
w_{p}=0 & & \text { on } \partial \Omega .
\end{aligned}\right.
$$

In the case of a convex $\Omega$ (where the shift theorem $\left\|w_{p}\right\|_{2} \leq c\left\|e_{p}\right\|_{0}$ holds),

$$
\begin{aligned}
\left\|e_{p}\right\|_{0}^{2} & =\left(e_{p},-\Delta w_{p}\right)=\left(\nabla e_{p}, \nabla w_{p}\right) \\
& =\left(\nabla e_{p}, \nabla\left(w_{p}-z_{p}\right)\right) \leq\left\|e_{p}\right\|_{1}\left\|\nabla\left(w_{p}-z_{p}\right)\right\|_{0} \\
& \leq c\left\|e_{p}\right\|_{1} p^{-1}\left\|w_{p}\right\|_{2} \leq c\left\|e_{p}\right\|_{1} p^{-1}\left\|e_{p}\right\|_{0}
\end{aligned}
$$

holds for some $z_{p} \in S^{p}$. So,

$$
\left\|e_{p}\right\|_{0} \leq c p^{-1}\left\|e_{p}\right\|_{1}
$$

and (2.4) follows. (Cf. [12, Prop. 3.2] for a similar argument for higher-order problems.) In the case of a nonconvex $\Omega$ partitioned with multiple elements, 
we split $w_{p}$-as done in [13] -according to (cf. (1.3))

$$
w_{p}=\bar{w}_{p}+\sum_{j=1}^{M} \sum_{\lambda_{j, l}<1} \gamma_{j, l} S_{j, l}
$$

with $\bar{w}_{p} \in H^{2}(\Omega)$, and

$$
\left\|\bar{w}_{p}\right\|_{2}+\sum_{\lambda_{j, l}<1}\left|\gamma_{j, l}\right| \leq c\left(\left\|e_{p}\right\|_{0}+\left\|w_{p}\right\|_{1}\right) \leq c\left\|e_{p}\right\|_{0}
$$

Thus, for some $z_{p} \in S^{p}$,

$$
\left\|\nabla\left(w_{p}-z_{p}\right)\right\|_{0} \leq c\left(p^{-1}\left\|\bar{w}_{p}\right\|_{2}+\sum_{\lambda_{j, l}<1}\left|\gamma_{j, l}\right| p^{-2 l \alpha_{j}}\right) \leq c p^{-1}\left\|e_{p}\right\|_{0}
$$

So, again, $\left\|e_{p}\right\|_{0} \leq c p^{-1}\left\|e_{p}\right\|_{1}$ and (2.4) follows.

3. THE $L^{2}$-DISTANCE OF THE $H_{0}^{1}$-PROJECTION FROM THE EXACT SOLUTION BOUNDED IN TERMS OF A STABILITY CONSTANT

Let $u_{p}$ be the Galerkin solution (i.e., $H_{0}^{1}$-projection onto $S^{p}$ ). We shall derive some lower bounds on the error $u-u_{p}$ measured in $L^{2}$, which are of lesser convergence rate than those of the $L^{2}$-projection. We recall that, cf. [3], for a problem like (1.1) with leading singularity $r^{\alpha} \sin \alpha \theta$,

$$
\inf _{v \in S^{p}}\|u-v\|_{0} \leq c p^{-(2 \alpha+2)}
$$

Following an idea of Wahlbin [17], one may easily prove

Proposition 3.1. Let $u$ and $u_{p}$ be the exact and discrete solutions to (1.1) as defined before. Let $\Omega$ have reentrant corners. Then, if the coefficient of the leading term $r^{\alpha} \sin \alpha \theta$ in the singular expansion of $u$ is nonzero, there exists a $c>0$ such that

$$
\left\|u-u_{p}\right\|_{0} \geq c p^{-4 \alpha} \text {. }
$$

Proof. A mere transcription of the main idea in [17]. Like in [17], it is enough to prove the result for a particular solution $u^{0}=a_{0} r^{\alpha} \sin \alpha \theta+\cdots, a_{0} \neq 0$. Let $K$ be an element in the triangulation of $\Omega$ with a vertex in common with a vertex of $\Omega$ ( $A_{M}$, say) that supports the leading singularity. Let $x_{0} \in \stackrel{\circ}{K}$ and let $B_{0} \subset \subset B_{1} \subset \subset K$ be disks centered at $x_{0}$ so that $\mathscr{A}_{0}=B_{1} \backslash B_{0}$ is an annulus. Let $\omega \in C^{\infty}(\bar{\Omega})$ be such that

$$
\omega= \begin{cases}1 & \text { outside } B_{1} \\ 0 & \text { inside } B_{0}\end{cases}
$$

and $G\left(x ; x_{0}\right)$ be the Green's function for $(1.1)$. Now let

$$
u^{0}(x)=\omega(x) G\left(x ; x_{0}\right)
$$

Then, $u^{0}$ has a singularity at the vertex of the type $r^{\alpha} \sin \alpha \theta$, as was shown in [17]. Let the $H_{0}^{1}$-projection of $u^{0}$ onto $S^{p}$ be denoted by $u_{p}^{0}$, and let 
$e_{p}^{0}=u^{0}-u_{p}^{0}$. Then, noticing that $\operatorname{supp}\left(\Delta u^{0}\right) \subset K$, we have

$$
\begin{aligned}
c p^{-4 \alpha} & \leq\left\|e_{p}^{0}\right\|_{1}^{2}=\int_{\Omega} \nabla e_{p}^{0} \nabla\left(u^{0}-u_{p}^{0}\right) d x \\
& =\int_{\Omega} \nabla e_{p}^{0} \nabla u^{0} d x=-\int_{\Omega} e_{p}^{0} \Delta u^{0} d x \\
& \leq\left\|e_{p}^{0}\right\|_{0, K}\left\|\Delta u^{0}\right\|_{0, K},
\end{aligned}
$$

yielding (3.2).

Remark 3.1. The estimate (3.2) yields a stricter lower bound than (3.1) for $\alpha<1$, i.e., the case of a reentrant corner. Based on the lower bound (3.2), we see that the estimate (2.4) is optimal in rate for the case of a crack domain. For other corner domains, the estimate $(2.4)$ is generally not sharp, as will be seen from the consideration below and numerical results later.

We next look at the convex domain case and assume for simplicity that $\Omega$ is a single parallelogram. We will then look at the situation where there is only one element, namely, $\Omega$ itself. Let $S^{p}=Q_{p}(F(\Omega)) \cap H_{0}^{1}(\Omega)$, where $F$ is affine, $\operatorname{det}(F)>0, F(\Omega)=(-1,1)^{2}$. Then we may integrate $(2.2)$ by parts to get

$$
-\int_{\Omega} u_{p} \Delta v d x=\int_{\Omega} f v d x, \quad \forall v \in S^{p} .
$$

We may alternatively formulate (3.3) as our finite element method with an asymmetric bilinear form. In this setting, an $L^{2}$-norm error estimate is the "natural" one for $e_{p}$, and this is intimately connected with the inf-sup or stability constant for the bilinear form in (3.3).

Denote, for convenience, the bilinear form by

$$
B(u, v)=-\int_{\Omega} u \Delta v d x, \quad u \in L^{2}, v \in H^{2} \cap H_{0}^{1}
$$

We introduce two constants,

$$
\begin{aligned}
& C(u)=\frac{\left\|u-u_{p}\right\|_{0}}{\inf _{v \in S^{p}}\|u-v\|_{0}}, \quad \text { the optimality constant at } u, \\
& D(u)=\sup _{v \in S^{p}} \frac{\left\|(u+v)_{p}\right\|_{0}}{\|u+v\|_{0}}, \quad \text { the stability constant at } u,
\end{aligned}
$$

where $u$ is given by $(1.1), u_{p}$ by $(3.3)$ and $(u+v)_{p} \in S^{p}$ by

$$
B\left((u+v)_{p}, w\right)=B(u+v, w), \quad \forall w \in S^{p}
$$

i.e., the projection of $u+v$ onto $S^{p}$ given by the inner product defined in (3.4). It was shown in [2] that $C$ and $D$ are interconnected,

$$
D(u)-1 \leq C(u) \leq D(u)+1 .
$$




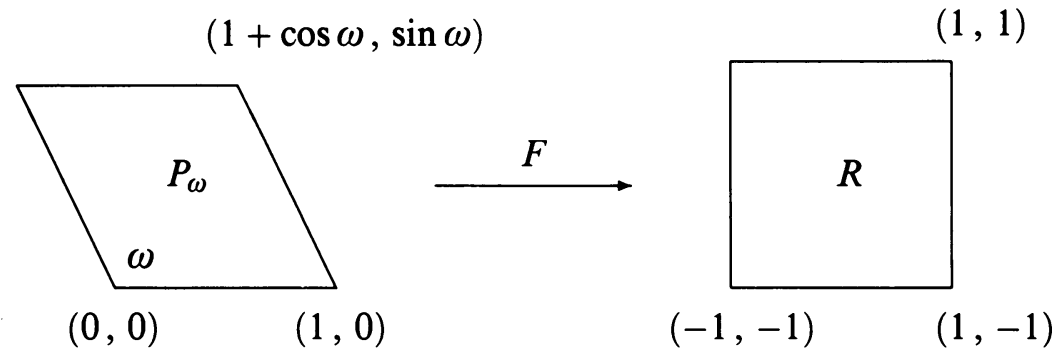

FIGURE 3.1. Affine map $F$ with pre-image and image depicted

The constant $D$ turns out to be connected to the inf-sup constant. We introduce two new constants,

$$
\begin{aligned}
& \mu=\inf _{u \in L^{2}} \sup _{v \in H^{2} \cap H_{0}^{1}} \frac{|B(u, v)|}{\|u\|_{0}\|v\|_{2}}, \\
& \mu_{p}=\inf _{u \in S^{p}} \sup _{v \in S^{p}} \frac{|B(u, v)|}{\|u\|_{0}\|v\|_{2}} .
\end{aligned}
$$

From the Buniakowsky-Cauchy-Schwarz inequality, we get

$$
|B(u, v)| \leq \sqrt{2}\|u\|_{0}\|v\|_{2},
$$

so that, via [2], the following relations hold,

$$
\frac{\mu}{\mu_{p}} \leq \sup _{u} D(u) \leq \frac{\sqrt{2}}{\mu_{p}} .
$$

We note that for any $u \in L^{2}(\Omega)$ there is a unique $v \in H^{2}(\Omega) \cap H_{0}^{1}(\Omega)$ such that $\Delta v=u$, and since $\Omega$ is convex, we have the estimate $\|v\|_{2} \leq c_{\Omega}\|u\|_{0}$. With (3.8), we easily get

$$
\frac{1}{c_{\Omega}} \leq \mu \leq \sqrt{2}
$$

Remark 3.2. Since $\Omega$ is convex, $\|v\|_{2}$ is equivalent to $\|\Delta v\|_{0}$ on $H^{2}(\Omega) \cap$ $H_{0}^{1}(\Omega)$. If we replace $\|v\|_{2}$ by $\|\Delta v\|_{0}$ in the definition of $\mu$, then we have $\mu=1$.

Clearly, the existence of quasi-optimal error estimates is linked with the infsup constant $\mu_{p}$. We will show that in some sense to be made more clear in the next lemma, it is enough to consider $\mu_{p}$ for a square.

Let $P_{\omega}$ be the parallelogram in Figure 3.1.

The affine mapping which maps $P_{\omega}$ onto $R=(-1,1)^{2}$ is

$$
F(x)=2\left(\begin{array}{cc}
1 & -\cot \omega \\
0 & \csc \omega
\end{array}\right) x-\left(\begin{array}{l}
1 \\
1
\end{array}\right) \text {. }
$$

Let $S_{\omega}^{p}=\left\{\tilde{v}=v \circ F: v \in S^{p}(R)\right\}$ with

$$
S^{p}(R)=\stackrel{\circ}{Q}_{p}(R)=\left(1-x_{1}^{2}\right)\left(1-x_{2}^{2}\right) Q_{p-2}(R) .
$$


Define, as in (3.7),

$$
\mu_{p, \omega}=\inf _{\tilde{u} \in S_{\omega}^{p}} \sup _{\tilde{v} \in S_{\omega}^{p}} \frac{|B(\tilde{u}, \tilde{v})|}{\|\tilde{u}\|_{0, P_{\omega}}\|\tilde{v}\|_{2, P_{\omega}}} .
$$

Lemma 3.2. Let $\mu_{p}=\mu_{p, \pi / 2}$ be as defined in (3.11) with respect to $P_{\pi / 2}=R$ and $\mu_{p, \omega}$ as in (3.11). Then, given $a \omega_{0} \in(0, \pi / 2)$, there exist $c$ and $C$, depending on $\omega_{0}$, so that

$$
c \mu_{p} \leq \mu_{p, \omega} \leq C \mu_{p}, \quad \forall \omega \in\left[\omega_{0}, \pi-\omega_{0}\right] .
$$

Proof. We prove the equivalence between the respective inner products in (3.11). Let $x \approx y$ denote an equivalence in the sense that there exist positive constants $c$ and $C$ independent of $x$ and $y$, so that $c x \leq y \leq C x$. First we see that

$$
\int_{P_{\omega}} \tilde{u} \tilde{v} d x=\int_{R} u v|J|^{-1} d x \approx \int_{R} u v d x,
$$

where $J=D F$ is the Jacobian of $F$ and $|J|=4 \csc \omega$, provided we require $\omega_{0} \leq \omega \leq \pi-\omega_{0}$ for some $\omega_{0} \in(0, \pi / 2)$. Secondly, one may prove that

$$
\int_{P_{\omega}} \nabla_{\tilde{x}} \tilde{u} \nabla_{\tilde{x}} \tilde{v} d x=\int_{R} \nabla u J^{T} J \nabla v|J|^{-1} d x \approx \int_{R} \nabla u \nabla v d x
$$

by verifying that the eigenvalues of $J^{T} J$ lie in a positive interval for $\omega_{0} \leq \omega \leq$ $\pi-\omega_{0}$. This gives the equivalence of the bilinear forms. Thirdly,

$$
|\tilde{u}|_{H^{2}\left(P_{\omega}\right)}^{2}=\int_{R}\left(D^{2} u\right)^{T} H D^{2} u|J|^{-1} d x \approx|u|_{H^{2}(R)}^{2},
$$

where $H=\sum_{i, j=1}^{2} f_{i j}^{T} f_{i j}$ with $f_{i j}=\left(J_{1 i} J_{1 j}, J_{1 i} J_{2 j}+J_{2 i} J_{1 j}, J_{2 i} J_{2 j}\right)$ and $D^{2} u=$ $\left(u_{11}, u_{12}, u_{22}\right)$. Through a lengthy calculation, it can be shown that the eigenvalues of $H$ are in some positive interval for $\omega_{0} \leq \omega \leq \pi-\omega_{0}$. We proved $|\tilde{u}|_{H^{k}\left(P_{\omega}\right)}^{2} \approx|u|_{H^{k}(R)}^{2}$ for $k=0,1$ earlier. Thus, $\|\tilde{u}\|_{H^{2}\left(P_{\omega}\right)}^{2} \approx\|u\|_{H^{2}(R)}^{2}$ and (3.12) follows from the definition (3.11).

Remark 3.3. We may equally well handle a reduction from an (oblique) triangle to a right-angled one.

Remark 3.4. If $\Omega \in \mathscr{P}$, then $\mu_{p} \geq c p^{-1}$ for some constant $c$ independent of $p$, by the equivalence in the above lemma and the lower bound in (3.9). For certain special cases involving $C^{1}$-elements, one will not be able to obtain a better inequality, $\mu_{p} \geq c p^{-\nu}$, for $\nu \in(0,1)$. Specifically, consider solving $-\Delta u=f$ in the V-shaped domain of Figure 6.1, with smooth $f$ and zero Dirichlet boundary conditions, using $p$-version $C^{1}$-elements. Collecting several earlier results, we have

$$
c(\alpha) p^{-4 \alpha} \leq\left\|u-u_{p}\right\|_{0} \leq C(\alpha)\left(\frac{\sqrt{2}}{\mu_{p}}+1\right) p^{-2 \alpha-2} .
$$

Now select $\alpha=(1+\varepsilon) / 2$ for small $\varepsilon>0$; then isolating $\mu_{p}$ and contracting the above inequalities, one finds

$$
\mu_{p} \leq \bar{c}(\varepsilon) p^{-1+\varepsilon} .
$$


Remark 3.5. Lemma 3.2 is significant in the sense that in the guaranteed upper estimate $C(u) \leq \sqrt{2} / \mu_{p, \omega}+1 \leq c / \mu_{p, \pi / 2}+1$, one will observe the same degree of suboptimality for all angles in any compact subinterval of $(0, \pi)$.

The inf-sup constant $\mu_{p, \pi / 2}$, which by Lemma 3.2 characterizes the behavior of all convex, single-element cases, can be computed numerically for a given choice of finite element space, via a generalized eigenvalue problem. This was done for $\stackrel{\circ}{Q}_{p}$ in [13] with an emerging behavior of

$$
c p^{-1 / 2} \leq \mu_{p, \pi / 2} \leq C p^{-1 / 2},
$$

indicating the loss of a half power of $p$ relative to the $L^{2}$-distance (3.1). It is an open problem to prove (3.13) theoretically. If one were able to show (3.13), it would follow that $\left\|e_{p}\right\|_{0} \leq c p^{-2 \alpha-3 / 2}$. We shall further justify this for a purely radial version of the problem in the next section and further demonstrate that the classical duality argument can be extended to obtain the optimal convergence estimate $\left\|e_{p}\right\|_{0} \leq c p^{-2 \alpha-3 / 2}$, in contrast to (2.4).

\section{A ONE-Dimensional ANALOGUE}

It is well known that the singularities of (1.1) supported at the vertices of $\Omega$ are essentially radial and of the form $r^{\alpha} g(r, \theta)$ in local polar coordinates. We shall investigate a purely radial model problem with such a singularity and $g \equiv 1$ for simplicity.

The purely radial singular function $u=r^{\alpha}, \alpha>0$, is the unique solution to

$$
\left\{\begin{array}{l}
\frac{1}{r}\left(r u^{\prime}\right)^{\prime}=\alpha^{2} r^{\alpha-2} \quad \text { in }(0,1), \\
|u(0)|<\infty, \quad u(1)=1 .
\end{array}\right.
$$

Let

$$
\mathscr{P}_{N}^{0}=\{v: v \text { is a polynomial of degree } \leq N, v(1)=0\} .
$$

Then, for $N \geq 1$, the Galerkin solution $u_{N} \in r+\mathscr{P}_{N}^{0}$ satisfies

$$
\int_{0}^{1}\left(u_{N}^{\prime}-u^{\prime}\right) v^{\prime} r d r=0, \quad \forall v \in \mathscr{P}_{N}^{0},
$$

which is then our simple one-dimensional problem.

Remark 4.1. An indication of how (4.1), then (4.3), could arise naturally follows. Let $\Omega$ be the intersection of a cone with opening $\pi / \alpha\left(\alpha \notin Z_{+}\right), \alpha \geq 1 / 2$, and the open unit ball centered at the apex of the cone whose right leg is assumed to coincide with the positive $x$-axis. Let $\tilde{u}=r^{\alpha} \sin \alpha \theta$ be the exact solution to

$$
\left\{\begin{array}{l}
-\Delta \tilde{u}=0 \text { in } \Omega=\{(r, \theta): r \in(0,1), \theta \in(0, \pi / \alpha)\}, \\
\tilde{u}=0 \text { on } \partial \Omega \cap\{(r, \theta): \theta=0 \text { or } \theta=\pi / \alpha\}, \\
\tilde{u}=\sin \alpha \theta \text { on } \partial \Omega \cap\{(r, \theta): r=1\} .
\end{array}\right.
$$

The purely radial part of $\tilde{u}$ is $u$. Suppose we used finite elements of the type $u_{N}(r) \sin \alpha \theta$, where $u_{N}(r)$ were a polynomial of degree at most $N$, equal to 1 
at $r=1$ (vanishing at 1 for the test functions). Then the Galerkin equation would read

$$
\begin{aligned}
0 & =\int_{\Omega} \nabla\left[\left(u_{N}-u\right) \sin \alpha \theta\right] \nabla[v \sin \alpha \theta] r d r d \theta \\
& =\frac{\pi}{2 \alpha} \int_{0}^{1}\left\{\left(u_{N}^{\prime}-u^{\prime}\right) v^{\prime}+\frac{\alpha^{2}}{r^{2}}\left(u_{N}-u\right) v\right\} r d r .
\end{aligned}
$$

If we now drop the zeroth-order term, we get (4.3).

Let us define the weighted inner product

$$
(u, v)=\int_{0}^{1} u v r d r
$$

for $u, v \in L_{01}^{2}(0,1)$, subscripts corresponding to our weight $(1-r)^{0} r^{1}$ at the endpoints of $(0,1)$. Then (4.3) implies:

Lemma 4.1. The derivative $u_{N}^{\prime}$ is the $L_{01}^{2}(0,1)$ projection of $u^{\prime}$ onto $\mathscr{P}_{N-1}$.

Proof. A constant is the derivative of a function in $\mathscr{P}_{N}^{0}: 1=d(r-1) / d r$. The remaining monomials of $\mathscr{P}_{N-1}$ also belong to $d\left(\mathscr{P}_{N}^{0}\right) / d r$.

Note that we may translate all statements to the interval $(-1,1)$ by the affine transformation

$$
(0,1) \ni r \mapsto x=t(r)=2 r-1 \in(-1,1) .
$$

We recall that on the standard interval $[-1,1]$ the Jacobi polynomials $P_{n}^{(\alpha, \beta)}(x), n \geq 0$, are orthogonal with respect to the weight $w(x)=$ $(1-x)^{\alpha}(1+x)^{\beta}, \alpha>-1, \beta>-1$. The value of $P_{n}^{(\alpha, \beta)}(x)$ at $x=1$ is $\left(\begin{array}{c}n+\alpha \\ n\end{array}\right)$, and

$$
\int_{-1}^{1} w(x)\left|P_{n}^{(\alpha, \beta)}(x)\right|^{2} d x=\frac{2^{\alpha+\beta+1}}{2 n+\alpha+\beta+1} \frac{\Gamma(n+\alpha+1) \Gamma(n+\beta+1)}{n ! \Gamma(n+\alpha+\beta+1)} .
$$

Also recall Rodrigues' formula

$$
P_{n}^{(\alpha, \beta)}(x)=\frac{(-1)^{n}}{2^{n} n !} \frac{1}{w(x)} \frac{d^{n}}{d x^{n}}\left[w(x)\left(1-x^{2}\right)^{n}\right] .
$$

We will use in particular the Jacobi polynomials $\left\{P_{n}^{(0,1)}\right\}_{n=0}^{\infty}$, which are orthogonal in $L_{01}^{2}(-1,1)$, cf. [6] or [16]. Let $\left\{j_{n}\right\}_{n=0}^{\infty}=\left\{P_{n}^{(0,1)} \circ t\right\}_{n=0}^{\infty}$ be the corresponding orthogonal sequence in $L_{01}^{2}(0,1)$ obtained via the transformation (4.7). Then, we have

Theorem 4.2. The following estimate holds for $u=r^{\alpha}$ :

$$
\left\|u^{\prime}-u_{N}^{\prime}\right\|_{L_{01}^{2}(0,1)} \leq c N^{-2 \alpha},
$$

and the estimate is sharp.

Proof. This follows from Lemma 4.1, the form of $u^{\prime}=\alpha r^{\alpha-1}$ and standard approximation results for polynomials, see [11], [8] or later [4]: using Rodrigues' 
and Stirling's formulas, one gets

$$
u^{\prime}=\sum_{n=0}^{\infty} b_{n} j_{n}, \quad u_{N}^{\prime}=\sum_{n=0}^{N-1} b_{n} j_{n},
$$

where

$$
\begin{aligned}
b_{n} & =\frac{\left(u^{\prime}, j_{n}\right)}{\left(j_{n}, j_{n}\right)}=(-1)^{n+1} 2(n+1) \frac{\Gamma(\alpha+1)}{\Gamma(-\alpha)} \frac{\Gamma(n+1-\alpha)}{\Gamma(n+2+\alpha)} \\
& =(-1)^{n+1} c_{0}(\alpha) n^{-2 \alpha}\left(1+(1+2 \alpha) / n+O\left(n^{-2}\right)\right)
\end{aligned}
$$

in which $c_{0}(\alpha)=\Gamma(\alpha+1) / \Gamma(-\alpha)$, and $\lim _{\alpha \rightarrow k} c_{0}(\alpha)=0$ for $k>0$ integer. We shall have occasion to use (4.10) again.

The same argument yields:

Proposition 4.3. The following $N$-distance holds for $u=r^{\alpha}$ in $L_{01}^{2}(0,1)$ with respect to the subspace $\mathscr{P}_{N}$ :

$$
\inf _{v \in \mathscr{P}_{N}}\|u-v\|_{L_{01}^{2}(0,1)}=c(\alpha) N^{-(2 \alpha+2)}\left(1+O\left(N^{-1}\right)\right)
$$

Remark 4.2. The results (4.8) and (4.11) give the "correct" rates also for the two-dimensional problems with dominant corner singularity $\tilde{u}$; this follows from the analysis in [3] and [7].

Let us calculate the $L_{01}^{2}(0,1)$ error estimate directly and show to what extent this in turn is provable via the usual duality technique. Define

$$
j_{n}^{(-1)}(r)=\int_{1}^{r} j_{n}(s) d s, \quad n \in Z_{+} \cup\{0\}
$$

Then, using (4.9), we get

$$
u-u_{N}=\sum_{n=N}^{\infty} b_{n} j_{n}^{(-1)},
$$

and it becomes beneficial to have a formula relating $j_{n}^{(-1)}$ to a linear combination of $j_{k}$.

Lemma 4.4. Let $n \geq 2$. Then the following formulas hold:

$$
j_{n}^{(-1)}=c_{n, n+1} j_{n+1}+c_{n, n} j_{n}+c_{n, n-1} j_{n-1},
$$

where

$$
\begin{gathered}
c_{n, n+1}=\frac{n+2}{(2 n+3)(2 n+2)}, \quad c_{n, n}=\frac{-1}{(2 n+3)(2 n+1)}, \\
c_{n, n-1}=\frac{-n}{(2 n+2)(2 n+1)}
\end{gathered}
$$

and

$$
\int_{1}^{x} P_{n}^{(0,1)}(u) d u=\bar{c}_{n, n+1} P_{n+1}^{(0,1)}(x)+\bar{c}_{n, n} P_{n}^{(0,1)}(x)+\bar{c}_{n, n-1} P_{n-1}^{(0,1)}(x),
$$

where, $\bar{c}_{n, k}=2 c_{n, k}, k=n+1, n, n-1$. 
Proof. By the substitution $u=2 s-1$,

$$
\begin{aligned}
j_{n}^{(-1)}(r) & =\int_{1}^{r} j_{n}(s) d s=\int_{1}^{r} P_{n}^{(0,1)}(2 s-1) d s \\
& =\frac{1}{2} \int_{1}^{2 r-1} P_{n}^{(0,1)}(u) d u,
\end{aligned}
$$

and we need only verify (4.16). Clearly,

$$
\int_{1}^{x} P_{n}^{(0,1)}(u) d u=\sum_{m=0}^{n+1} \bar{c}_{n, m} P_{m}^{(0,1)}(x)
$$

and we may integrate by parts to get with

$$
(u, v)=\int_{-1}^{1} u v(1+x) d x
$$

that

$$
\begin{aligned}
\bar{c}_{n, m}\left(P_{m}^{(0,1)}, P_{m}^{(0,1)}\right)=\sum_{k=0}^{n+1} \bar{c}_{n, k}\left(P_{m}^{(0,1)}, P_{k}^{(0,1)}\right) \\
=\int_{-1}^{1}\left(\int_{1}^{x} P_{n}^{(0,1)}(u) d u\right) P_{m}^{(0,1)}(x)(1+x) d x \\
=\left[\left(\int_{1}^{x} P_{n}^{(0,1)}(u) d u\right)\left(\int_{-1}^{x} P_{m}^{(0,1)}(u)(1+u) d u\right)\right]_{-1}^{1} \\
-\int_{-1}^{1} P_{n}^{(0,1)}(x)\left(\int_{-1}^{x} P_{m}^{(0,1)}(u)(1+u) d u\right) d x \\
=-\int_{-1}^{1} P_{n}^{(0,1)}(x)\left(\int_{-1}^{x} P_{m}^{(0,1)}(u)(1+u) d u\right) d x
\end{aligned}
$$

for $m \in Z_{+} \cup\{0\}$, by orthogonality. Here, we may factor

$$
\int_{-1}^{x} P_{m}^{(0,1)}(u)(1+u) d u=(1+x) q_{m+1}(x)
$$

for some $q_{m+1} \in \mathscr{P}^{m+1}$. Thus,

$$
\bar{c}_{n, m}=0 \quad \text { for } 0 \leq m \leq n-2 .
$$

Identity (4.16) in [16] specializes to

$$
(2 n+2) P_{n}^{(0,1)}(x)=(n+2) P_{n}^{(1,1)}(x)-(n+1) P_{n-1}^{(1,1)}(x)
$$

and identity (4.14) in [16] gives

$$
\frac{d}{d x} P_{n}^{(0,0)}(x)=\frac{1}{2}(n+1) P_{n-1}^{(1,1)}(x),
$$

so that, with $l_{n}(x)=P_{n}^{(0,0)}(x)$, the Legendre polynomial of degree $n$,

$$
P_{n}^{(0,1)}(x)=\frac{1}{n+1}\left(l_{n+1}^{\prime}(x)-l_{n}^{\prime}(x)\right) \text {. }
$$


Identity (4.16) in [16] also yields

$$
(1+u) P_{n}^{(0,1)}(u)=P_{n}^{(0,0)}(u)+P_{n+1}^{(0,0)}(u),
$$

so that

$$
\int_{-1}^{x} P_{m}^{(0,1)}(u)(1+u) d u=\int_{-1}^{x} l_{m}(u) d u+\int_{-1}^{x} l_{m+1}(u) d u .
$$

But, for $j \geq 1$,

$$
\begin{aligned}
\int_{-1}^{1} l_{i}^{\prime}(x) & \left(\int_{-1}^{x} l_{j}(u) d u\right) d x \\
& =\left[l_{i}(x)\left(\int_{-1}^{x} l_{j}(u) d u\right)\right]_{-1}^{1}-\int_{-1}^{1} l_{i}(x) l_{j}(x) d x \\
& =-\frac{2}{2 i+1} \delta_{i j} .
\end{aligned}
$$

Substituting (4.20) and (4.22) into (4.18), using (4.23) and the fact that $\left(P_{m}^{(0,1)}, P_{m}^{(0,1)}\right)=2 /(m+1)$, we have

$$
\begin{aligned}
& \bar{c}_{n, n+1}=\frac{n+2}{(2 n+3)(n+1)}, \\
& \bar{c}_{n, n}=-\frac{2}{(2 n+3)(2 n+1)}, \\
& \bar{c}_{n, n-1}=-\frac{n}{(2 n+1)(n+1)} .
\end{aligned}
$$

Combining (4.17), (4.19), and (4.24) yields (4.16).

Inserting (4.14) into (4.13) gives

$$
\begin{aligned}
u-u_{N}= & \sum_{n=N}^{\infty} b_{n}\left(c_{n, n+1} j_{n+1}+c_{n, n} j_{n}+c_{n, n-1} j_{n-1}\right) \\
= & \sum_{k=N+1}^{\infty}\left(c_{k-1, k} b_{k-1}+c_{k, k} b_{k}+c_{k+1, k} b_{k+1}\right) j_{k} \\
& +\left(c_{N, N} b_{N}+c_{N+1, N} b_{N+1}\right) j_{N}+c_{N, N-1} b_{N} j_{N-1} .
\end{aligned}
$$

Since $\left|c_{k, k}\right|=1 /\left(4 k^{2}\right)+O\left(k^{-3}\right)$ and $-c_{k+1, k}$ and $c_{k-1, k}$ are both equal to $1 /(4 k)+O\left(k^{-2}\right)$, we get

$$
\begin{aligned}
\left\|u-u_{N}\right\|_{L_{01}^{2}}^{2} & =c\left\{\sum_{k=N+1}^{\infty} k^{-4 \alpha} k^{-3} k^{-1}+N^{-4 \alpha} N^{-2} N^{-1}\right\}\left(1+O\left(N^{-1}\right)\right) \\
& =c N^{-4 \alpha-3}\left(1+O\left(N^{-1}\right)\right),
\end{aligned}
$$

so that

$$
\left\|u-u_{N}\right\|_{L_{01}^{2}}=c N^{-2 \alpha-3 / 2}\left(1+O\left(N^{-1}\right)\right) .
$$


This is half a power off the $N$-distance in (4.11). We will now show that the usual duality argument can be extended to recover (4.26) and not just one power better than the $H^{1}$ estimate (4.8).

Remark 4.3. One notes that the last two terms in (4.25) dominate asymptotically, cf. Lemma 5.2 .

Theorem 4.5. Let $u=r^{\alpha}$ and $u_{N}$ be determined by (4.3). Then there exist positive constants independent of $N$ so that

$$
c N^{-(2 \alpha+3 / 2)} \leq\left\|u-u_{N}\right\|_{L_{01}^{2}} \leq C N^{-(2 \alpha+3 / 2)} .
$$

Proof. This is a restatement of (4.26). We will prove the second inequality once more, now via an extension of the usual duality argument, in the hope of providing a possible approach to deal with higher-dimensional problems. Let $e_{N}=u-u_{N}$. Then, defining $w_{N}$ by

$$
\left\{\begin{array}{l}
-\frac{1}{r}\left(r w_{N}^{\prime}\right)^{\prime}=e_{N} \quad \text { in }(0,1), \\
\left|w_{N}(0)\right|<\infty, \quad w_{N}(1)=0,
\end{array}\right.
$$

we get for any $z_{N} \in \mathscr{P}_{N}^{0}$,

$$
\begin{aligned}
\left(e_{N}, e_{N}\right) & =\int_{0}^{1} e_{N}\left(-\frac{1}{r}\left(r w_{N}^{\prime}\right)^{\prime}\right) r d r=-\left[e_{N}\left(r w_{N}^{\prime}\right)\right]_{0}^{1}+\int_{0}^{1} e_{N}^{\prime} w_{N}^{\prime} r d r \\
& =\left(e_{N}^{\prime}, w_{N}^{\prime}-z_{N}^{\prime}\right)=\left(e_{N},-\frac{1}{r}\left(r\left(w_{N}^{\prime}-z_{N}^{\prime}\right)\right)^{\prime}\right) \\
& =\left(e_{N}, e_{N}+\frac{1}{r}\left(r z_{N}^{\prime}\right)^{\prime}\right) \leq\left\|e_{N}\right\|_{L_{01}^{2}}\left\|e_{N}+\frac{1}{r}\left(r z_{N}^{\prime}\right)^{\prime}\right\|_{L_{01}^{2}}
\end{aligned}
$$

by repeated integration by parts and the $H_{0}^{1}$-projection nature of $u_{N}$. An immediate corollary is

$$
\left\|e_{N}\right\|_{L_{01}^{2}}=\inf _{z_{N} \in \mathscr{P}_{N}^{0}}\left\|e_{N}+\frac{1}{r}\left(r z_{N}^{\prime}\right)^{\prime}\right\|_{L_{01}^{2}} .
$$

One gets a similar result for a single element in two dimensions. Now $\mathscr{P}_{N-2}$ is contained in the image of $\mathscr{P}_{N}^{0}$ under the map $z \mapsto(1 / r)\left(r z^{\prime}\right)^{\prime}$, and we get from (4.9) and (4.11), and by using Lemma 4.4, that

$$
\begin{aligned}
\left\|e_{N}+\frac{1}{r}\left(r z_{N}^{\prime}\right)^{\prime}\right\|_{L_{01}^{2}} \leq & \inf _{\xi_{N-2} \in \mathscr{P}_{N-2}}\left\|u-\xi_{N-2}\right\|_{L_{01}^{2}}+\inf _{\zeta_{N-2} \in \mathscr{P}_{N-2}}\left\|u_{N}-\zeta_{N-2}\right\|_{L_{01}^{2}} \\
\leq & c N^{-2 \alpha-2}+\inf _{\zeta_{N-2} \in \mathscr{P}_{N-2}}\left\|\sum_{n=0}^{N-1} b_{n} j_{n}^{(-1)}-\zeta_{N-2}\right\|_{L_{01}^{2}} \\
\leq & c N^{-2 \alpha-2}+\left\|b_{N-1} c_{N-1, N} j_{N}\right\|_{L_{01}^{2}} \\
& +\left\|\left(b_{N-2} c_{N-2, N-1}+b_{N-1} c_{N-1, N-1}\right) j_{N-1}\right\|_{L_{01}^{2}} \\
\leq & c N^{-(2 \alpha+3 / 2)} .
\end{aligned}
$$


Remark 4.4. These results do not follow automatically from [2] or [8], since we are dealing with an entirely different inner product geared towards the twodimensional setting. They could, on the other hand, be used to establish instability results like the ones in [2], now for the nonconstant coefficient operator $-\frac{1}{r} \frac{d}{d r}\left(r \frac{d}{d r}\right)$.

Remark 4.5. It is not difficult to extend the results to a three-dimensional model scenario with the operator $-\frac{1}{r^{2}} \frac{d}{d r}\left(r^{2} \frac{d}{d r}\right)$, or model $d$-dimensional problems with symmetry group $S^{d-1}$.

Remark 4.6. It is interesting that the Green's function no longer has the $r^{\alpha}$ singularity used to prove (3.2) in Proposition 3.1, but that we may still prove a Wahlbin-like lower bound along the same lines (with $u^{0}=r^{\alpha}$ ) to obtain

$$
\left\|e_{N}\right\|_{L_{01}^{2}} \geq c \frac{\sqrt{2 \alpha-2}}{\alpha^{2}} N^{-4 \alpha},
$$

which is valid only for $\alpha \geq 1$ (i.e. corresponding to the convex case).

\section{SHARP $L^{\infty}$-ERROR ESTIMATES FOR THE ONE-DIMENSIONAL MODEL}

To better understand how far one can go with error estimates for the $p$ version method, we derive various $L^{\infty}$-norm error estimates for $p$-version finite element solutions for the one-dimensional model problem considered in the previous section. We need asymptotic expansions of the various coefficients that appeared.

Lemma 5.1. For the coefficients defined in (4.15), we have

$$
\begin{aligned}
& c_{n, n+1}=\frac{1}{4 n}-\frac{1}{8 n^{2}}+O\left(n^{-3}\right), \\
& c_{n, n}=-\frac{1}{4 n^{2}}+O\left(n^{-3}\right), \\
& c_{n, n-1}=-\frac{1}{4 n}+\frac{3}{8 n^{2}}+O\left(n^{-3}\right), \\
& c_{n-1, n}=\frac{1}{4 n}+\frac{1}{8 n^{2}}+O\left(n^{-3}\right), \\
& c_{n+1, n}=-\frac{1}{4 n}+\frac{5}{8 n^{2}}+O\left(n^{-3}\right) .
\end{aligned}
$$

The proof of the lemma is elementary, and hence is omitted.

Now let us define

$$
\begin{aligned}
& d_{N, k}=c_{k-1, k} b_{k-1}+c_{k, k} b_{k}+c_{k+1, k} b_{k+1}, \quad k \geq N+1, \\
& d_{N, N}=c_{N, N} b_{N}+c_{N+1, N} b_{N+1}, \\
& d_{N, N-1}=c_{N, N-1} b_{N} .
\end{aligned}
$$

Then, the error expression (4.25) can be rewritten as

$$
u-u_{N}=\sum_{k=N+1}^{\infty} d_{N, k} j_{k}+d_{N, N} j_{N}+d_{N, N-1} j_{N-1} .
$$


For the asymptotic behavior of $\left\{d_{N, k}\right\}$, we have

Lemma 5.2. There holds

$$
\begin{aligned}
& d_{N, k}=(-1)^{k}(1+\alpha) c_{0}(\alpha) k^{-2 \alpha-2}\left(1+O\left(k^{-1}\right)\right), \quad k \geq N+1, \\
& d_{N, N}=(-1)^{N+1} \frac{c_{0}(\alpha)}{4} N^{-2 \alpha-1}\left(1+O\left(N^{-1}\right)\right), \\
& d_{N, N-1}=(-1)^{N} \frac{c_{0}(\alpha)}{4} N^{-2 \alpha-1}\left(1+O\left(N^{-1}\right)\right) .
\end{aligned}
$$

The proof of Lemma 5.2 can be made by combining the results from Lemma 5.1 and (4.10).

Now, we are ready to give $L^{\infty}$-norm error estimates on $u-u_{N}$.

Theorem 5.3. Let $\alpha \notin Z_{+}$. Then there exists a positive $c$ so that

$$
\left\|u-u_{N}\right\|_{L^{\infty}(0,1)} \leq c N^{-2 \alpha},
$$

and the estimate is sharp.

Proof. From [1, Formula 22.14.1], we have

$$
\left|P_{n}^{(0,1)}(x)\right| \leq n+1, \quad x \in[-1,1] .
$$

Thus,

$$
\left|j_{n}(r)\right| \leq n+1, \quad r \in[0,1] .
$$

Then, from (5.4) and Lemma 5.2, we get

$$
\begin{aligned}
\left|u-u_{N}\right| \leq & \sum_{k=N+1}^{\infty} c_{0}(\alpha)(1+\alpha) k^{-2 \alpha-1}\left(1+O\left(k^{-1}\right)\right) \\
& +\frac{c_{0}(\alpha)}{4} N^{-2 \alpha}\left(1+O\left(N^{-1}\right)\right)+\frac{c_{0}(\alpha)}{4} N^{-2 \alpha}\left(1+O\left(N^{-1}\right)\right) \\
\leq & c(\alpha) N^{-2 \alpha} .
\end{aligned}
$$

To show that the estimate is sharp, we compute the error at $r=0$. We have

$$
j_{n}(0)=P_{n}^{(0,1)}(-1)=(-1)^{n}(n+1) .
$$

Hence,

$$
\begin{aligned}
u(0)-u_{N}(0)= & \sum_{k=N+1}^{\infty}(-1)^{k}(1+\alpha) c_{0}(\alpha) k^{-2 \alpha-2}\left(1+O\left(k^{-1}\right)\right)(-1)^{k}(k+1) \\
& +(-1)^{N+1} \frac{c_{0}(\alpha)}{4} N^{-2 \alpha-1}\left(1+O\left(N^{-1}\right)\right)(-1)^{N}(N+1) \\
& +(-1)^{N} \frac{c_{0}(\alpha)}{4} N^{-2 \alpha-1}\left(1+O\left(N^{-1}\right)\right)(-1)^{N-1} N \\
= & (1+\alpha) c_{0}(\alpha) \sum_{k=N+1}^{\infty} k^{-2 \alpha-1}\left(1+O\left(k^{-1}\right)\right) \\
& -\frac{c_{0}(\alpha)}{2} N^{-2 \alpha}\left(1+O\left(N^{-1}\right)\right) \\
= & \frac{1}{2 \alpha} c_{0}(\alpha) N^{-2 \alpha}+O\left(N^{-2 \alpha-1}\right),
\end{aligned}
$$


where we have used the fact that

$$
\sum_{k=N+1}^{\infty} k^{-2 \alpha-1}=\frac{1}{2 \alpha} N^{-2 \alpha}\left(1+O\left(N^{-1}\right)\right),
$$

which is evident from the following inequalities:

$$
\int_{N+1}^{\infty} t^{-2 \alpha-1} d t \leq \sum_{k=N+1}^{\infty} k^{-2 \alpha-1} \leq \int_{N}^{\infty} t^{-2 \alpha-1} d t
$$

Thus, as long as $\alpha \notin Z_{+}$(which excludes the trivial cases of smooth monomial solutions), the estimate $(5.5)$ is sharp.

It is a well-known phenomenon in error estimations of finite element solutions that usually the order of an $L^{\infty}$-norm error estimate is almost the same as that of an $L^{2}$-norm error estimate. Comparing the above result with Theorem 4.5, we expect we can get higher-order error estimates in an $L^{\infty}$-like norm which is compatible with the $L_{01}^{2}$ norm. Let us introduce a weighted $L^{\infty}$ space,

$$
L_{01}^{\infty}(0,1)=\left\{u \text { measurable }:\|u\|_{L_{01}^{\infty}(0,1)}=\text { ess } \sup _{r \in(0,1)}|r u(r)|<\infty\right\} .
$$

Theorem 5.4. Let $\alpha \notin Z_{+}$. Then there exists a positive constant $c$ such that

$$
\left\|u-u_{N}\right\|_{L_{01}^{\infty}(0,1)} \leq c N^{-2 \alpha-3 / 2},
$$

and the estimate is sharp. As a consequence, we have a superconvergence resuli

$$
\max _{r \in I}\left|u(r)-u_{N}(r)\right| \leq c(I) N^{-2 \alpha-3 / 2}
$$

for any closed interval $I \subset(0,1]$.

Proof. From (4.21), we get

$$
r j_{n}(r)=\left(l_{n}(2 r-1)+l_{n+1}(2 r-1)\right) / 2 .
$$

Thus, by using (5.4), we find

$$
r\left(u(r)-u_{N}(r)\right)=\sum_{k=N+1}^{\infty} d_{N, k} r j_{k}(r)+d_{N, N} r j_{N}(r)+d_{N, N-1} r j_{N-1}(r)=E_{1}+E_{2},
$$

where

$$
\begin{aligned}
& E_{1}=\frac{1}{2}\left\{\sum_{k=N+2}^{\infty}\left(d_{N, k}+d_{N, k-1}\right) l_{k}(2 r-1)\right. \\
& \left.\quad+d_{N, N+1} l_{N+1}(2 r-1)+\left(d_{N, N}+d_{N, N-1}\right) l_{N}(2 r-1)\right\}, \\
& E_{2}=\frac{1}{2}\left\{d_{N, N} l_{N+1}(2 r-1)+d_{N, N-1} l_{N-1}(2 r-1)\right\} .
\end{aligned}
$$

From Lemma 5.2, we find

$$
\begin{aligned}
& d_{N, k}+d_{N, k-1}=O\left(k^{-2 \alpha-3}\right), \quad k \geq N+2, \\
& d_{N, N+1}=O\left(N^{-2 \alpha-2}\right), \\
& d_{N, N}+d_{N, N-1}=O\left(N^{-2 \alpha-2}\right) .
\end{aligned}
$$


Thus,

$$
\left|E_{1}\right| \leq c N^{-2 \alpha-2} .
$$

To deal with the term $E_{2}$, we use the following two identities (cf. [1]):

$$
\begin{gathered}
l_{n}(x)-l_{n+1}(x)=(1-x) P_{n}^{(1,0)}(x), \\
P_{n}^{(1,0)}(x)+P_{n+1}^{(1,0)}(x)=\frac{n+3 / 2}{n+1}(1+x) P_{n}^{(1,1)}(x) .
\end{gathered}
$$

From (5.7) and (5.8), we find that

$$
l_{n}(x)-l_{n+2}(x)=\frac{n+3 / 2}{n+1}(1-x)(1+x) P_{n}^{(1,1)}(x) .
$$

From Formula 8.965 in [9, page 1037], we have

$$
(1-x)^{3 / 4}(1+x)^{3 / 4} P_{n}^{(1,1)}(x)=g(x) n^{-1 / 2}+O\left(n^{-3 / 2}\right)
$$

for some bounded, nonzero function $g(x)$. Using (5.9), we then have

$$
l_{n}(x)-l_{n+2}(x)=\frac{n+3 / 2}{n+1}(1-x)^{1 / 4}(1+x)^{1 / 4} g(x) n^{-1 / 2}+O\left(n^{-3 / 2}\right) .
$$

Therefore, we have, again using Lemma 5.2,

$$
\begin{aligned}
E_{2} & =(-1)^{N} \frac{c_{0}}{8} N^{-2 \alpha-1}\left(l_{N-1}(2 r-1)-l_{N+1}(2 r-1)\right)+O\left(N^{-2 \alpha-2}\right) \\
& =\tilde{g}(r) N^{-2 \alpha-3 / 2}+O\left(N^{-2 \alpha-2}\right),
\end{aligned}
$$

where $\tilde{g}$ is a bounded, nonzero function. Thus, the theorem is proved.

Similarly, from the expansion for the error $u^{\prime}-u_{N}^{\prime}$, we have the following results. We omit the proofs of these results.

Theorem 5.5. If $\alpha>1$, we have the error estimate

$$
\left\|u^{\prime}-u_{N}^{\prime}\right\|_{L^{\infty}(0,1)} \leq c N^{-2 \alpha+2}
$$

and the estimate is sharp. When $\alpha<1$, there is no convergence of $u_{N}^{\prime} \rightarrow u^{\prime}$ in $L^{\infty}(0,1)$.

Remark 5.1. It follows that the $W^{1, \infty}$-stability holds: $\left\|u_{N}\right\|_{W^{1, \infty}(0,1)} \leq$ $c\|u\|_{W^{1, \infty}(0,1)}$, if $\alpha>1$.

Theorem 5.6. There holds

$$
\left\|u^{\prime}-u_{N}^{\prime}\right\|_{L_{01}^{\infty}(0,1)} \leq c N^{-2 \alpha-1 / 2},
$$

and the estimate is sharp. As a consequence, for any closed interval $I \subset(0,1]$, we have

$$
\max _{r \in I}\left|u^{\prime}(r)-u_{N}^{\prime}(r)\right| \leq c(I) N^{-2 \alpha-1 / 2}
$$




\section{NUMERICAL EXPERIMENTS}

We report the numerical results of a two-dimensional problem related to (1.1), with the same sort of majorizing singularity, but with Neumann boundary conditions imposed weakly in order to guarantee a simple numerical implementation of the boundary conditions for an exact solution

$$
u=r^{\alpha} \sin (\alpha \theta) \text {. }
$$

Consider the V-shaped domain, $\Omega$, depicted in Figure 6.1.

In our figure, $\alpha=2 / 3$, but we will vary $\alpha$ in the interval $\left(\frac{1}{2}, 1\right)$. We then use the $p$-version of the Galerkin method with merely one element $\left(=\frac{1}{2} \Omega\right)$ to compute numerical solutions to the problem indicated in Figure 6.2.

If we let $\partial \Omega^{+}$denote the union of those three line segments bounding $\frac{1}{2} \Omega$ on which we do not have $u=0$ imposed, then we may define the numerical solution, $u_{N} \in V_{N}$, to be

$$
\int_{\frac{1}{2} \Omega} \nabla u_{N} \cdot \nabla v d x=\int_{\partial \Omega_{+}} g v d \gamma, \quad \forall v \in V_{N},
$$

where $V_{N}=W_{N} \circ F$. Here, $F$ is the affine mapping defined in Figure 3.1 and

$$
W_{N}=\underset{\substack{i=0, \ldots, p \\ j=1, \ldots, p}}{\operatorname{span}}\left\{L_{i}(s) L_{j}(t)\right\}
$$

We use here integrals of the Legendre polynomials, $L_{i}(\xi)=\int_{-1}^{\xi} P_{i-1}^{(0,0)}(\eta) d \eta$, for $i \geq 1$ and $L_{0}(\xi)=1$, in order to have convenient analytical expressions for the Dirichlet forms and $L^{2}$ inner products acting on pairs of basis functions (leading to sparse stiffness and mass matrices). The load (the boundary integrals involving $g_{i}$ ) and the errors are computed using Simpson quadrature with 600 nodes.

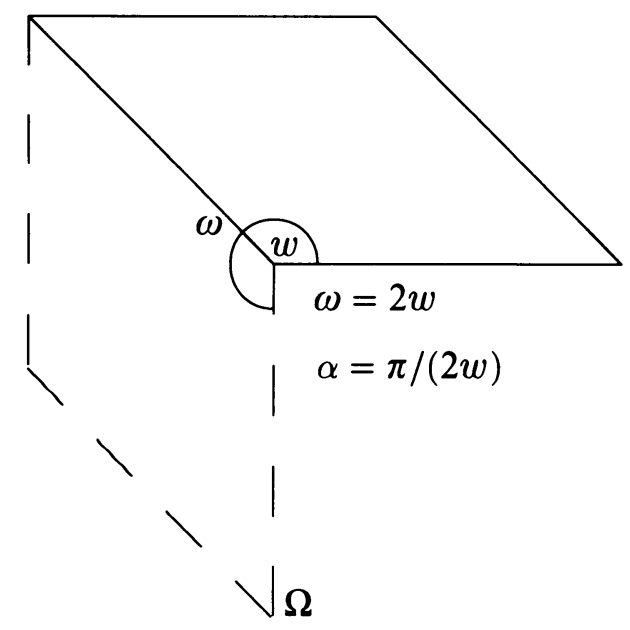

FIGURE 6.1. V-shaped domain $\Omega$ with reentrant corner of angle $2 w$ 


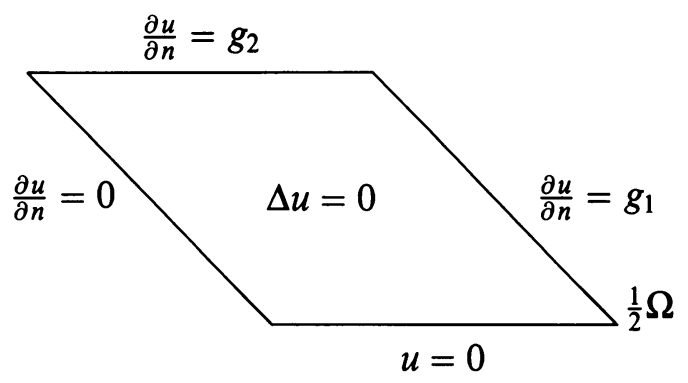

FIGURE 6.2. Elliptic boundary value problem posed over $\frac{1}{2} \Omega$

We have computed up to $p=20$; the observed rates of convergence in the $L^{2}$ norm are depicted below:

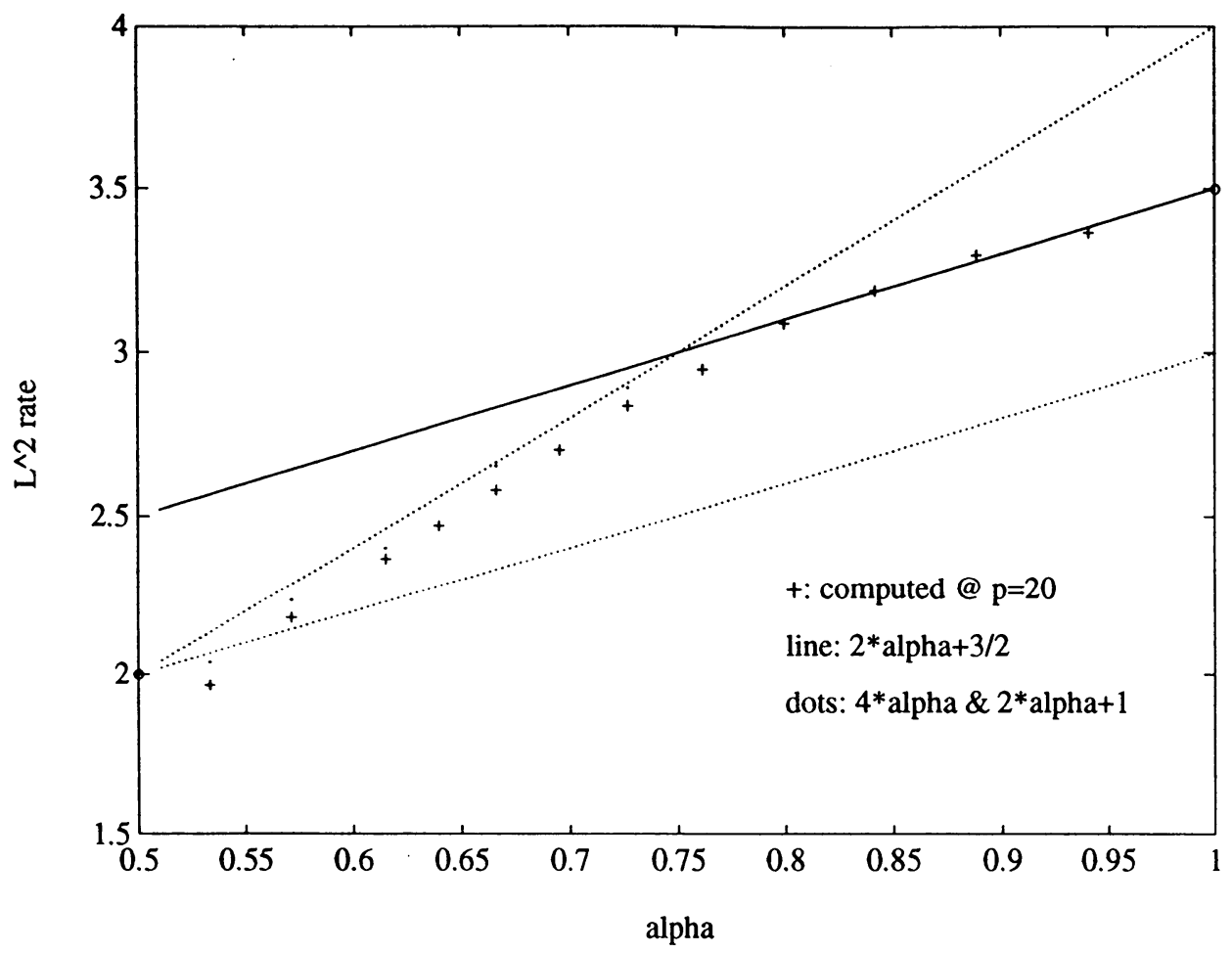

FIGURE 6.3. Numerically observed $L^{2}$ rate of convergence as dependent on $\alpha$

The rates of convergence in $H^{1}$ all confirm the known rate of $-2 \alpha$. Although the numerical results for $L^{2}$ are somewhat inconclusive, there is some indication that a conjectured rate of $-\min \{4 \alpha, 2 \alpha+3 / 2\}$, when the corner singularity is predominant, would be worth a proof. 


\section{ACKNOWLEDGMENT}

The authors thank two anonymous referees for their valuable comments leading to improvements in the presentation of the paper and for pointing out a mistake in an asymptotic expansion formula in an earlier version of the manuscript.

\section{BIBLIOGRAPHY}

1. M. Abramowitz, and I. A. Stegun (eds.), Handbook of mathematical functions, Dover, New York, 1972.

2. D. N. Arnold, I. Babuška, and J. Osborn, Finite element methods: Principles for their selection, Comput. Methods Appl. Mech. Engrg. 45 (1984), 57-96.

3. I. Babuška and $\mathrm{M}$. Suri, The optimal convergence rate of the p-version of the finite element method, SIAM J. Numer. Anal. 24 (1987), 750-776.

4. C. Bernardi and Y. Maday, Polynomial approximation of some singular functions, Publ. du Lab. d'Anal. Numér. R 90017, Univ. de Pierre et Marie Curie, Paris, 1990.

5. M. Dauge, S. Nicaise, M. Bourlard, and J.M.-S. Lubuma, Coefficients des singularités pour des problèmes aux limites elliptiques sur un domaine à points coniques II: Quelques opérateurs particuliers, Modél. Math. Anal. Numér. 24 (1990), 343-367.

6. P. J. Davis and P. Rabinowitz, Methods of numerical integration, 2nd ed., Academic Press, Orlando, 1984.

7. M. Dorr, The approximation theory for the p-version of the finite element method, SIAM J. Numer. Anal. 21 (1984), 1180-1207.

8. K. Eriksson, Some error estimates for the p-version of the finite element method, SIAM J. Numer. Anal. 23 (1986), 403-411.

9. I. S. Gradshteyn and I. M. Ryzhik, Tables of integrals, series and products, Academic Press, New York, 1975.

10. P. Grisvard, Elliptic problems in nonsmooth domains, Pitman, Boston, 1985.

11. W. Gui and I. Babuška, The $h, p$ and $h-p$ versions of the finite element method in 1 dimension: Part 1. The error analysis of the p-version, Numer. Math. 49 (1986), 577-612.

12. S. Jensen, On computing the pressure by the $p$ version of the finite element method for Stokes problem, Numer. Math. 59 (1991), 581-601.

13. S. Jensen and M. Suri, On the $L^{2}$-error of the p-version of the finite element method, Comput. Methods Appl. Mech. Engrg. 97 (1992), 233-243.

14. R.B. Kellogg, Higher order singularities for interface problems, The Mathematical Foundations of the Finite Element Method (A.K. Aziz, ed.), Academic Press, New York, 1972, pp. 589-602.

15. V.A. Kondrat'ev, Boundary value problems for elliptic equations in domains with conical or angular points, Trans. Moscow Math. Soc. 16 (1967), 227-313.

16. F.G. Tricomi, Vorlesungen über Orthogonalreihen, 2nd corr. ed., Grundlehren Vol. 76, Springer, Berlin, 1970.

17. L.B. Wahlbin, On the sharpness of certain local estimates for $\stackrel{\circ}{H^{1}}$ projections into finite element spaces: influence of a reentrant corner, Math. Comp. 42 (1984), 1-8.

Department of Mathematics, University of Iowa, Iowa City, Iowa 52242

E-mail address: whan@math. uiowa.edu

Department of Mathematics, University of Maryland, Baltimore County, BaltiMORE, MARYLAND 21228

E-mail address: jensen@math.umbc.edu 